\title{
Dream Recall Frequency and Sleep in Patients with Rapid Eye Movement Sleep Behavior Disorder
}

\author{
Min Jae Seong ${ }^{1}$, A-reum Jung ${ }^{1}$, Hea Ree Park ${ }^{1}$, Su Jung Choi ${ }^{2}$, Eun Yeon Joo ${ }^{3}$ \\ ${ }^{1}$ Department of Neurology, Samsung Medical Center, Sungkyunkwan University School of Medicine, Seoul, \\ ${ }^{2}$ Department of Nursing, Samsung Medical Center, Department of Clinical Nursing Science, Sungkyunkwan University School of Medicine, \\ Seoul, \\ ${ }^{3}$ Department of Neurology, Brain-Nerve Center, Samsung Medical Center, Samsung Biomedical Research Institute (SBRI), \\ Sungkyunkwan University School of Medicine, Seoul, Korea
}

\author{
렘수면행동장애 환자의 꿈 회상 빈도와 수면의 질 \\ 성민재 ${ }^{1}$, 정아름 ${ }^{1}$, 박혜리 $^{1}$, 최수정 ${ }^{2}$, 주은연 $^{3}$ \\ 성균관대학교 의과대학 삼성서울병원 신경과, ${ }^{1}$ 삼성서울병원 간호부, 성균관대학교 임상간호대학원, ${ }^{2}$ \\ 성균관대학교 의과대학 신경과학교실, 삼성서울병원 뇌신경센터 ${ }^{3}$
}

$\begin{array}{ll}\text { Received } & \text { November 29, } 2017 \\ \text { Revised } & \text { December 19, } 2017 \\ \text { Accepted } & \text { December 20, } 2017\end{array}$

Address for correspondence

Eun Yeon Joo, MD, PhD

Department of Neurology,

Brain-Nerve Center,

Samsung Medical Center,

Samsung Biomedical Research

Institute (SBRI), Sungkyunkwan

University School of Medicine,

81 Irwon-ro, Gangnam-gu,

Seoul 06351, Korea

Tel: +82-2-3410-3599

Fax: +82-2-3410-0052

E-mail: ejoo@skku.edu
Objectives: The dream recall and sleep of patients with rapid eye movement sleep behavior disorder (RBD) were not sufficiently studied. We hypothesized that RBD patients have frequent dream recall with poor sleep quality, and investigated the relationship between the dream recall frequency and sleep quality in RBD patients compared to controls. Methods: We analyzed 81 drug naïve patients [RBD (+), 64.6 \pm 8.3 y, 57 males] and 81 age and gender matched patients with sleep disturbances without RBD [RBD (-), $63.7 \pm 7.3$ y, 57 males]. All completed Pittsburgh sleep quality index (PSQI), insomnia severity index (ISI), Epworth sleepiness scale and Beck depression inventory. The 5-point rating scale was used to categorize dream recall frequency of most recent month ( $0=$ never, $4=$ very frequent). Results: In $\mathrm{RBD}(+)$, dream recall frequency was much higher [frequent dreaming, $77.2 \%$ vs. $35.4 \%$ ], and subjective sleep quality was much better [PSQI, 6.36 \pm 3.26 vs. 8.71 \pm 4.69 ]. Insomnia severity was much less in RBD (+) (ISI, 9.13 \pm 5.86$)$ than RBD (-) (12.43 \pm 7.62$)$. No significant differences were found in sleep parameters except lower N2 sleep \% in RBD $(+)$. The relationship between dream recall frequency and sleep was not significant in RBD (+), yet, a positive correlation was noted in RBD (-). Conclusions: RBD (+) had better sleep quality despite higher frequency of dream recall compared to RBD (-). Also dream recall was not related to their sleep quality in RBD (+), which suggests that RBD patients may have different sleep perception about their sleep and sleep quality.

J Sleep Med 2017;14(2):55-60

\section{서 론}

렘수면행동장애(rapid eye movement sleep behavior disorder, RBD)는 렘수면 동안 정상적으로 발생하는 골격근의 무긴장증(atonia)이 소실되어 꿈 내용을 행동화하는 질환이 다. ${ }^{1}$ 렘수면행동장애는 일반적으로 50 대 이상의 중장년층에

This is an Open Access article distributed under the terms of the Creative Commons Attribution Non-Commercial License (http://creativecommons.org/licenses/by-nc/4.0) which permits unrestricted non-commercial use, distribution, and reproduction in any medium, provided the original work is properly cited.
서 발생하며 남자에서 빈도가 높다. ${ }^{2}$

$\mathrm{RBD}$ 는 신경퇴행성 질환인 파킨슨병(Parkinson's disease), 루이소체 치매(dementia with Lewy bodies), 다발성 신경계 위축증(multiple system atrophy) 등과 관련성이 높으며 특 히 파킨슨병의 전구 질환으로 보고하는 연구가 많다. ${ }^{3-5}$

$\mathrm{RBD}$ 환자의 꿈은 난폭하고 생생하며 싸우거나 도망치는 내용이 특징이다. ${ }^{1,6}$ 일반적으로, 난폭하거나 동물이 등장하는 꿈은 성인보다는 소아에서 더 높은 빈도로 관찰된다. ${ }^{7,8}$ 연령 이 증가할수록 위협 회피 기술(threat-avoidance skill)이 향 
상함에 따라 이러한 내용의 꿈은 빈도가 감소한다. ${ }^{7}$ 하지만 신경퇴행성 질환인 $\mathrm{RBD}$ 는 퇴행성 과정을 통해 피질 활성도 (cortical activity), 신경심리학적인 측면, 자율신경 및 후각 기능의 감소가 발생하여 생물 개체 발생학적인(ontogenetically) 초기의 꿈의 양상을 갖게 된다고 추정한다. 9.10

꿈은 렘수면 기간 동안 각성 상태와 유사한 대뇌 활동에 의 한 내용을 피질 각성(cortical arousal)을 통해 기억을 하게 되 어 깨어난 후 회상하면서 인지하게 된다. ${ }^{11}$ 따라서 꿈의 빈도 에 대한 연구에 따르면 꿈을 많이 꾸는 사람은 잦은 피질 각 성에 의해 수면의 질이 낮은 것으로 알려져 있다. ${ }^{12,13}$

$\mathrm{RBD}$ 환자들은 꿈을 많이 꾼다고 호소한다. 그러나 $\mathrm{RBD}$ 환 자에서 꿈 회상 빈도에 대해서는 연구된 바가 거의 없다. RBD 환자들의 주관적인 수면의 질에 대해서도 알려진 바가 적다.

본 연구진들은, 신경퇴행성 과정을 겪는 $\mathrm{RBD}$ 환자들은 질 병의 초기일지라도 $\mathrm{RBD}$ 를 동반하지 않는 대조군에 비해 꿈 회상 빈도가 유의하게 높고, 잦은 꿈은 환자의 수면의 질에 영향을 줄 것으로 가정했다. 이를 증명하기 위하여 약물을 쓰지 않는 특발성 RBD 환자를 모집하여 대조군과의 꿈 회상 빈도와 수면의 질을 비교하고, 꿈과 수면지표 및 다른 임상 지표와의 관련성을 확인하고자 하였다.

\section{방 법}

\section{피험자}

2014년부터 2017년 11월까지 삼성서울병원에서 야간 수면 다원검사(overnight polysomnography, PSG) 및 수면 설문지 를 시행한 대상에서 $\mathrm{RBD}$ 로 진단받은 81명과 연령 및 성별 을 맞춘 RBD 외 다른 수면 문제로 내원하였거나 연구목적 으로 검사를 시행한 정상군을 포함한 81명을 대상으로 하였 다. 연령은 50대 이상으로 제한하였다. 대조군의 환자 분포는 폐쇄성 수면무호흡증 환자가 42명(51.9\%), 정상군으로 지원 했으나, 수면의 질 설문지에서 5 점 이상[Pittsburgh sleep quality index(PSQI) $\geq 5$ ] 측정된 주관적 수면장애 환자 39명 (48.1\%)이었다. RBD 진단은 미국수면의학회 국제수면질환 분류 제3판(American association of sleep medicine, international classification of sleep disorders-III)에 근거하였다. $\mathrm{RBD}$ 환자군 중 이차성 $\mathrm{RBD}(\mathrm{sec}$ ndary $\mathrm{RBD}$ )와 약물을 복용 중인 대상자는 제외하였다. $\mathrm{RBD}$ 환자군에서 우울 또는 불안 으로 정신과적 치료를 받는 환자는 없었다.

본 연구에 사용된 모든 연구 기준과 방법 및 평가는 삼성서 울병원 임상윤리심의위원회의 임상시험윤리심의를 획득하 였으며, 임상윤리심의위원회의 관리감독하에 시행되었다 (2017-11-055).

\section{야간 수면다원검사(Overnight polysomnography)}

PSG 장비는 RemLogic(Embla System, Broomfield, CO, $\mathrm{USA}$ )을 이용하였다. 수면의 단계 및 각성의 판정은 6채널 뇌 파(C3-A2, C4-A1, F3-A2, F4-A1, O1-A2, O2-A1), 4채널 안전도(electro-oculogram) 및 턱 근전도(chin electromyography)로 하였고, 호흡량은 온도감지센서(thermistor), 비압 측 정기(nasal pressure transducer), 호흡운동은 흥곽 및 복부벨 트(thoracic \& abdominal plethysmograph belts)로 측정하 였으며 산소포화도(finger pulse oximetry), 근전도는 늑간근 근전도 및 양쪽 전경골근에서 측정하였고 심전도(modified V2 lead)도 측정하였다. 폐쇄성 수면무호흡(obstructive sleep apnea)은 호흡운동이 유지된 상태에서 호흡이 10초 이상 $90 \%$ 이상 감소를 보인 경우로 정의하였고, 저호흡(hypopnea)은 호흡량 진폭이 10 초 이상 $30 \%$ 이상 감소를 보이면서 산소포 화도가 $4 \%$ 이상 감소하거나, 각성이 동반되는 경우로 정의하 였다. ${ }^{14}$ 수면 한 시간당 발생하는 폐쇄성 무호흡과 저호흡 발 생 횟수를 무호흡-저호흡지수(apnea-hypopnea index, AHI) 로 정의하였고 무호흡증의 중증도 지표로 사용하였다. $\mathrm{AHI}$ 가 5 이상인 경우 무호흡증으로 진단하였으며 경증 $(\mathrm{AHI}<15)$, 중등도 $(15 \leq \mathrm{AHI}<30)$, 중증 $(\mathrm{AHI} \geq 30)$ 으로 분류하였다. ${ }^{15}$ 렘수면무긴장소실(rapid eye movement sleep without atonia) 은 2012년 미국수면학회 판독 기준에 따라 진단했다. 렘수면 시의 한 에포크(epoch)를 30초로 정하였고, 이를 3초씩 구분 하여 미니 에포크를 지정했다. 기존 비렘수면 시의 최소 진폭 (amplitude)보다 증가된 턱근전도상의 근긴장이 한 에포크 의 $50 \%$ 이상에서 연속적으로 증가되어 나타날 경우 긴장성 (tonic) 렘수면무긴장소실[rapid eye movement(REM) sleep without atonia]로 정의하였다. 렘수면 시 미니 에포크를 기 준으로 5 개 이상에서 기존 배경 턱이나 양측 전경골근의 근전 도 진폭에 비해 0.1 5초의 기간 동안 최소 4배 이상 증가된 경 우, 위상성(phasic)의 렘수면무긴장소실로 정의하였다..$^{14}$ 연 구에 포함된 대상의 PSG 및 수면 설문지를 후향적으로 분 석하였다.

\section{설문지}

모든 피험자에서 PSG 검사 당일, 취침 전에 설문지를 시행 하였다. 생활습관에는 음주, 흡연, 카페인 섭취, 수면제 섭취 에 대한 항목이 있으며 동반 질환 및 복용하는 약물도 기록하 게 한다. 수면-각성 습관은 취침시간, 총 수면시간, 수면잠복 기, 기상시간을 기록한다. 잠꼬대, 이갈이, 코골이, 수면무호 흡 병력도 확인한다.

꿈 회상 빈도는 '지난 한 달간 얼마나 자주 꿈을 꾸었는가?' 라는 질문에 대한 5단계(5-point rating scale)로 답변하게 한 
다 $(0=$ 전혀 없음, $4=$ 매우 자주). 꿈의 빈도는 범주형 분석을 하기 위해 $0,1,2$ 는 드물게 꿈을 꾼다로, 3,4 는 자주 꿈을 꾼 다는 것으로 분류하였다(Supplementary Materials in the online-only Data Supplement).

주관적인 수면의 질은 PSQI로 평가하였으며, 불면증은 insomnia severity index(ISI), 주간 졸림은 Epworth sleepiness scale(ESS) 및 우울은 Beck depression inventory(BDI) 로 측정하였다. 인지기능에 대한 평가는 시행하지 않았다.

\section{통계 방법}

자료의 정규분포에 따라 통계 방법을 선택하였다. 연속변수 는 t-test 또는 정규분포를 만족하지 않는 경우 Mann-Whitney test로 분석하였고 평균과 표준편차를 구하였다. 범주형 변수는 Fisher의 정확한 검정(Fisher's exact test) 또는 카이 제곱검정 (chi-squared test)으로 통계분석을 시행하였다. 꿈 의 빈도와 수면의 질 간의 상관관계는 Spearman 상관분석 (Spearman's correlation analysis)을 했다. 통계적 유의성은 $p$ 값 0.05 미만을 기준으로 하였다. 통계분석에는 PASW Statistics for Windows, Version 18.0(SPSS Inc., Chicago, IL, USA)을 사용하였다.

\section{결 과}

총 81 명의 $\mathrm{RBD}(+)$ 군의 평균 연령은 $64.68 \pm 8.36$ 세였으며 57 명 $(70.4 \%)$ 이 남성이었다. 81 명의 $\mathrm{RBD}(-)$ 군의 평균 연령은
$63.73 \pm 7.37$ 세였으며 57명(70.4\%)이 남성으로 양 군 간의 차 이는 없었다. 음주, 카페인 섭취, 운동, 동반 질환 유무도 양 군 간 차이가 없었다(Table 1).

꿈 회상 빈도는 $\mathrm{RBD}(+)$ 군에서 뚜렷하게 높았다[RBD(+) 61 명(77.2\%) vs. $\mathrm{RBD}(-)$ 28명(35.4\%), $p<0.001]$. 잠꼬대 또한 $\mathrm{RBD}(+)$ 군에서 통계적으로 많았다 $[\mathrm{RBD}(+)$ 76명(95\%) vs. $\mathrm{RBD}(-)$ 36명(48.9\%), $p<0.001$ ](Table 1).

주관적 수면의 질[PSQI; $\mathrm{RBD}(+) 6.36 \pm 3.26 \mathrm{vs} . \mathrm{RBD}(-)$ $8.71 \pm 4.69, p<0.001]$ 과 불면증 정도[ISI; $\mathrm{RBD}(+)$ 9.13 \pm 5.86 vs. $\mathrm{RBD}(-) 12.43 \pm 7.62, p=0.003]$ 는 $\mathrm{RBD}(-)$ 군에서 유의하게 높았다(Table 1).

수면제 복용비율도 $\mathrm{RBD}(-)$ 군에서 의미 있게 높았다[RBD (+) 6명(8.1\%) vs. $\mathrm{RBD}(-)$ 22명(28.9\%), $p=0.001]$. 주간 졸음 $(\mathrm{ESS})$ 과 우울감(BDI)은 두 군 간 차이가 없었다(Table 1).

수면다원검사의 지표 중 두 군 간 차이를 보였던 것은 비 렘수면 2단계 수면비율이었으며 $[R B D(+) ~ 52.77 \pm 11.59 \%$ vs. $\mathrm{RBD}(-) 56.99 \pm 11.52 \%, p=0.02]$, 수면잠복기, 수면호흡 사건, 입면 후 각성, 수면효율, 각성지수 및 주기성사지운동지수 등 은 통계적인 차이가 없었다(Table 2).

$\mathrm{RBD}(+)$ 군은 꿈 회상 빈도와 수면의 질을 포함한 다른 임 상요인과의 관련성을 보기 위한 Spearman correlation 분석 에서는 유의미한 결과가 관찰되지 않았다. REM 수면 비율 과 꿈 회상 빈도도 관련성을 보이지 않았다 $(\mathrm{r}=0.13, p=0.09)$. 반면, $\mathrm{RBD}(-)$ 군에서는 꿈 회상 빈도와 우울감(BDI; $r=0.26$, $p=0.01$, 불면(ISI; $\mathrm{r}=0.30, p=0.007)$, 주관적인 수면의 질(PSQI;

Table 1. Demographics, life style and habitual sleep questionnaire of REM sleep behavior disorder $(+)(n=81)$ and REM sleep behavior disorder $(-)(n=81)$

\begin{tabular}{lccc}
\hline \multicolumn{1}{c}{ Parameters } & $\mathrm{RBD}(+)(\mathrm{n}=81)$ & $\mathrm{RBD}(-)(\mathrm{n}=81)$ & $p$ value \\
\hline Age, years & $64.68 \pm 8.36$ & $63.73 \pm 7.37$ & 0.44 \\
Gender-male, n (\%) & $57(70.4)$ & $57(70.4)$ & 1.00 \\
Alcohol consumption & $37(47.4)$ & $31(39.2)$ & 0.30 \\
Caffeine consumption & $53(68.8)$ & $48(61.5)$ & 0.34 \\
Exercise & $41(53.2)$ & $31(40.8)$ & 0.12 \\
Combined disease & $53(68.8)$ & $46(66.7)$ & 0.78 \\
Taking sleep pill & $6(8.1)$ & $22(28.9)$ & $0.001^{*}$ \\
Sleep talking & $76(95.0)$ & $36(48.9)$ & $<0.001^{*}$ \\
Bruxism & $13(17.8)$ & $12(16.0)$ & 0.76 \\
Dreaming frequency (frequent) & $28(35.4)$ & $<0.001^{*}$ \\
PSQI & $61(77.2)$ & $8.71 \pm 4.69$ & $<0.001^{*}$ \\
ESS & $6.36 \pm 3.26$ & $7.51 \pm 5.11$ & 0.44 \\
BDI & $8.09 \pm 4.35$ & $15.74 \pm 9.51$ & $12.43 \pm 7.62$ \\
ISI & $13.60 \pm 7.64$ & 0.12 \\
\hline
\end{tabular}

${ }^{*} p<0.05$. REM: rapid eye movement, PSQI: Pittsuburgh sleep quality index, ESS: Epworth sleepiness scale, BDI: Beck depression inventory, ISI: insomnia severity index, RBD: REM sleep behavior disorder 
Table 2. Polysomnographic data of RBD $(+)(n=81)$ and RBD (-) $(n=81)$

\begin{tabular}{|c|c|c|c|}
\hline Parameters & $\operatorname{RBD}(+)(\mathrm{n}=81)$ & $\operatorname{RBD}(-)(\mathrm{n}=81)$ & $p$ value \\
\hline Time in bed, min & $450.79 \pm 54.92$ & $446.70 \pm 51.40$ & 0.62 \\
\hline Total sleep time, min & $395.22 \pm 412.47$ & $352.67 \pm 56.95$ & 0.35 \\
\hline Sleep latency, min & $17.62 \pm 24.53$ & $14.77 \pm 17.04$ & 0.39 \\
\hline REM latency, min & $106.53 \pm 67.78$ & $105.31 \pm 64.05$ & 0.90 \\
\hline \multicolumn{4}{|l|}{ Sleep stage, $\%$} \\
\hline Non REM & $78.97 \pm 7.53$ & $80.82 \pm 7.42$ & 0.11 \\
\hline N1 sleep & $24.01 \pm 13.63$ & $21.98 \pm 12.77$ & 0.32 \\
\hline N2 sleep & $52.77 \pm 11.59$ & $56.99 \pm 11.52$ & $0.02^{*}$ \\
\hline Slow wave sleep & $2.21 \pm 3.71$ & $1.87 \pm 3.52$ & 0.55 \\
\hline REM sleep & $21.02 \pm 7.53$ & $18.90 \pm 7.64$ & 0.07 \\
\hline $\mathrm{RDI}, / \mathrm{h}$ & $23.38 \pm 21.26$ & $19.17 \pm 14.55$ & 0.14 \\
\hline AHI, /h & $19.57 \pm 18.28$ & $17.54 \pm 15.10$ & 0.44 \\
\hline REM AHI, /h & $23.08 \pm 25.81$ & $27.25 \pm 14.32$ & 0.40 \\
\hline REM RDI, /h & $23.78 \pm 22.35$ & $28.02 \pm 13.92$ & 0.25 \\
\hline REM arousal index, /h & $20.70 \pm 18.75$ & $20.11 \pm 9.75$ & 0.84 \\
\hline Wakefulness after sleep onset, \% & $19.00 \pm 14.87$ & $18.37 \pm 8.97$ & 0.74 \\
\hline Sleep efficiency, \% & $78.25 \pm 15.84$ & $79.01 \pm 9.63$ & 0.71 \\
\hline Arousal index, $/ \mathrm{h}$ & $22.89 \pm 11.95$ & $23.16 \pm 12.31$ & 0.88 \\
\hline PLM index, /h & $39.71 \pm 37.71$ & $28.77 \pm 40.16$ & 0.12 \\
\hline $\mathrm{MAI}, / \mathrm{h}$ & $2.77 \pm 4.22$ & $2.94 \pm 5.44$ & 0.83 \\
\hline Lowest $\mathrm{SaO}_{2}, \%$ & $87.98 \pm 4.88$ & $86.40 \pm 6.07$ & 0.07 \\
\hline ODI, /h & $14.94 \pm 16.89$ & $13.87 \pm 13.55$ & 0.65 \\
\hline
\end{tabular}

${ }^{*} p<0.05$. RBD: REM sleep behavior disorder, REM: rapid eye movement, RDI: respiratory disturbance index, AHI: apnea-hypopnea index, PLM: periodic limb movement, MAI: movement arousal index, ODI: oxygen desaturation index

Table 3. Spearman rho correlation of the dream recall frequency and the sleep quality of ESS, BDI, ISI, and PSQI in RBD (+) and $\operatorname{RBD}(-)$

\begin{tabular}{lcc}
\hline Parameters & RBD $(+)(\mathrm{n}=81)$ & $\mathrm{RBD}(-)(\mathrm{n}=81)$ \\
\hline ESS & $0.12(0.29)$ & $0.12(0.27)$ \\
BDI & $0.48(0.68)$ & $0.26(0.01)^{*}$ \\
ISI & $0.16(0.14)$ & $0.30(0.007)^{*}$ \\
PSQI & $0.01(0.94)$ & $0.37(0.001)^{*}$ \\
\hline
\end{tabular}

Displayed as 'Spearman's r. ${ }^{*} p<0.05$. ESS: Epworth sleepiness scale, BDI: Beck depression inventory, ISI: insomnia severity index, PSQI: Pittsuburgh sleep quality index, RBD: rapid eye movement sleep behavior disorder

$\mathrm{r}=0.37, p=0.001)$ 간에 양의 상관관계를 보였다(Table 3).

\section{고 찰}

본 연구는 RBD 환자의 인구학적 특성, 수면다원검사 소 견, 꿈의 빈도 및 수면의 질을 분석하고 대조군과 비교한 후 꿈 회상 빈도와 수면의 질 간의 상관성을 분석한 것이다.

\section{$\mathrm{RBD}$ 환자의 꿈 회상 빈도}

예상했던 대로, 꿈 회상 빈도는 $\mathrm{RBD}(+)$ 에서 훨씬 더 높은 빈도로 보고되었다. 꿈을 꾸는 기전으로 각성-회상 모델이 가장 많이 언급되는데, 이는 피질 각성(cortical arousal)을 통 해 장기 기억으로 정보가 옮겨지는 과정으로 설명하며, 이 과 정은 수면 중 각성이 필요하다고 한다. ${ }^{11}$ 따라서 꿈을 많이 꿀 수록 수면 중 각성의 증가에 따른 수면의 질 저하가 초래된다 고 한다. ${ }^{12,13} \mathrm{RBD}(+)$ 환자에서 꿈 회상 빈도가 현저히 많음 에도 불구하고, $\mathrm{RBD}(-)$ 군에 비해 각성지수나 수면 중 각성, 수면효율 등 수면의 질을 나타내는 지수가 더 나쁘지 않았으 며, 꿈의 빈도와 PSQI와의 상관성도 관찰되지 않았다. 오히 려 $\mathrm{RBD}(-)$ 군에서 꿈의 빈도와 $\mathrm{PSQI}$, 불면 정도 및 우울 정 도와 유의한 상관성이 보였다.

한편, $\mathrm{RBD}$ 환자의 꿈 회상 빈도의 증가는 꿈의 특성에 의 하며 RBD의 특징인 생생하고 움직임이 많은 꿈의 내용과 꿈 의 행동화로 인한 부상으로 인해 꿈 회상이 증가한다고 보고 한 바가 있다. ${ }^{9}$ 각성-인출 모델(arousal-retrieval model)을 통한 회상 과정에서 돌출(salience)과 간섭(interference)이 
일어나고, 감정적 변화와 인상적인 꿈 내용은 돌출을 거쳐 회상을 강화시키는 것으로 추정했다. ${ }^{11}$

$\mathrm{RBD}$ 환자의 생생한 꿈 회상도 단순한 피질 각성에 의한 것보다는 꿈의 내용 특성에 따른 꿈의 회상의 증가로 추정해 볼 수 있다. 정상 소아는 꿈의 내용이 난폭하거나 동물이 등 장하는 꿈을 꾸기도 하는데, 이는 위협-자극계(threat-stimulation system)의 부적절한 활성화에서 시작한다고 설명한 다. ${ }^{7}$ 이런 나쁜 꿈은 소아가 성장하면서 저절로 없어지는데, $\mathrm{RBD}$ 환자에서 신경퇴행 과정이 진행되면서 도로 발생하는 것으로 받아들여지고 있다. 증가된 위상성 근전도의 활성화 를 환자의 과도한 운동과 동적 움직임이 많은 꿈의 원인으 로 설명하기도 한다. ${ }^{9}$

이전 $\mathrm{RBD}$ 의 연구에 따르면 $\mathrm{RBD}$ 환자에서 우울이 심한 경우 꿈 회상이 낮다는 보고를 하였다. ${ }^{16}$ 꿈은 감정을 포함하 고 있으며 감정적 조절장애(emotional dysregulation)가 있 는 경우는 꿈 회상이 감소한다고 보고하였으며 이러한 원인 으로 RBD 환자 내에서 우울감에 따른 꿈 회상의 빈도가 차 이를 보인다고 설명한다. ${ }^{17}$ 그러나 우리 연구에서는 두 군에 서 우울감의 차이는 없었으며, 따라서 꿈 회상의 빈도 차이 는 감정적 조절장애가 관여하기보다는 $\mathrm{RBD}$ 의 꿈의 내용과 관련이 있을 것으로 추측한다.

\section{$\mathrm{RBD}$ 환자의 긍정적 수면 상태 오인}

$\mathrm{RBD}$ 환자들의 인구학적 특성은 기존 연구(50대 이상, 남 성에서 호발) 결과와 대략 일치하였다. ${ }^{18} \mathrm{RBD}$ 환자들은 꿈 은 많다고 호소하지만, 불면증이나 기타 수면 자체에 대해 호 소하는 경우는 별로 없다. 그런데 수면다원검사에서는 수면 무호흡지수와 각성지수 증가, N3 수면 감소 등 객관적인 수 면의 질 저하가 뚜렷하게 관찰되었다.

본 환자들의 PSQI, ISI, ESS, BDI를 포함한 자기 보고 수 면 설문지 결과를 보면, 대조군에 비해 높은 수면 만족도와 낮 은 불면 상태를 보고했다. 주간졸음증은 심하지 않고, 우울감 은 약간 높은 상태이나, 대조군과 유의한 차이가 없었다. 이 는 기존의 연구결과와 조금 다르다. RBD 환자의 주간졸음 에 대한 한 연구에 따르면 $\mathrm{ESS}$ 가 대조군에 비해 높았는데, ${ }^{19}$ 다 른 연구에서는 정상인과 차이 없는 것으로 보고하여서 $\mathrm{RBD}$ 환자의 주간졸음 여부는 아직 일치된 의견이 없다..$^{18}$ 또한 동 일 연구에서 보고한 결과를 보면, RBD 환자에서 PSQI와 ISI 값이 유의하게 높아서, 본 연구결과와 상반된다. ${ }^{20}$ 기존 연구 는 $\mathrm{RBD}$ 를 진단받고 약물 치료 중인 환자가 포함되어 있고, 본 환자들은 모두 RBD 치료를 받기 전 상태였기 때문에 약 물 복용에 따른 수면의 질과 주간기능의 차이가 존재했을 것으로 추정하며 본 연구의 대조군 질환 분포 또한 이전 연
구와 다른 결과를 보인 이유로 생각된다. 이와 비슷한 예로, 폐쇄성 수면무호흡증(obstructive sleep apnea syndrome) 환 자의 수면의 질도 호흡 사건의 심각도와 뚜렷한 연관성이 없 다는 연구가 있다. ${ }^{19}$ 폐쇄성 수면무호흡증 환자의 수면 무호 흡-저호흡 지수(AHI)는 PSQI와 상관성을 보이지 않고 오 히려 저산소증이 수면의 질과 관련이 있다고 하였다. ${ }^{21}$ 산소 포화도가 경도로 저하된 경우에는 PSQI를 높게, 즉 수면의 질을 나쁘게 평가하지만, 산소포화도가 고도로 저하된 경우 에는 오히려 수면의 질을 높게 평가한다고 한다. ${ }^{21}$ 폐쇄성 수 면무호흡증의 고해상도-기능적 MRI 검사에서 전두엽, 측 두엽, 두정엽 및 앞쪽 띠이랑의 기능 저하가 관찰되는데, 이 영역의 기능적 손상이 인지기능 저하와 수면의 질 오인(misperception)을 유발한다고 추정한 바 있다. ${ }^{21}$ 따라서 본 연 구의 $\mathrm{RBD}$ 환자에서 객관적 지표는 명백한 수면장애를 나타 냄에도 불구하고, 주관적인 만족도가 높다는 것은 $\mathrm{RBD}$ 환 자들의 대뇌피질의 손상과 관련된 긍정적 수면 상태 오인 (positive sleep state misperception)의 일종일 가능성을 시 사한다..$^{22}$ 부정적 수면 상태 오인(negative sleep state misperception)은 뇌파상의 베타 또는 감마파의 증가와 관련 있 으며 이는 대뇌 활성화가 증가함에 따라 수면 중 기억상실 (mesograde amnesia)의 감소로 인한 부정적 수면 상태 오인 을 보이는 것으로 알려져 있다. 23,24 일반적으로 퇴행성 뇌질 환의 경우 뇌파는 베타파의 감소와 함께 전반적인 서파가 증가하며, $\mathrm{RBD}$ 또한 뇌파에서 서파가 증가되어 있는 것으로 알려져 있으며 25 이러한 기전으로 $\mathrm{RBD}$ 의 경우 긍정적 수면 오인 상태가 나타날 수 있을 것으로 생각된다.

\section{$\mathrm{RBD}$ 환자의 꿈 회상 빈도와 수면의 질 간의 상관성}

가설과 달리, 본 연구에서는 $\mathrm{RBD}(+)$ 환자들의 꿈 회상 빈 도와 수면의 질 및 임상요인 간의 상관성이 관찰되지 않았다. 오히려 $\mathrm{RBD}(-)$ 군에서 꿈 회상 빈도는 횔씬 적고 상관성이 낮긴 하지만, 불면, 우울감, 주관적 수면의 질과 유의한 상관 성을 보였다. 이는 아마도 대조군으로 사용한 $\mathrm{RBD}(-)$ 군에 불면증 환자군이 포함되어 있기 때문으로 추정한다. 불면과 우울감 빈도가 높은 불면증 환자에서 꿈 회상 비율도 함께 높음은 이미 잘 알려져 있다. ${ }^{26,27} \mathrm{RBD}$ 와 $\mathrm{RBD}$ 가 아닌 다른 수 면 질환 간의 꿈 회상 기전 차이에 의한 것으로 생각되며, 이 에 따라 $\mathrm{RBD}$ 외의 수면 질환에서는 피질 각성의 정도와 꿈 회상 빈도가 상관관계를 보이는 것으로 추정된다.

이전에도 $\mathrm{RBD}$ 환자의 수면의 질에 대한 연구가 드물게 있었으나 꿈 빈도와 관련하여 수면의 질에 접근한 연구는, 본 논문이 처음이다. 또한 본 연구에 포함된 환자군은 처음 질환을 진단받고 약물 치료를 시작하기 전의 대상자였다는 
점이 가장 큰 특징이다.

본 연구의 몇 가지 제한점은 다음과 같다. 첫째, 꿈 회상 빈도를 다섯 단계에 따라 나눈 후 꿈이 많은 군과 그렇지 않 은 군으로 범주화 분석을 시행한 점이다. 둘째, 꿈 내용에 대한 분석은 시행하지 않았다. 셋째, 대조군으로 사용한 $\mathrm{RBD}(-)$ 군 으로 수면호흡장애 환자가 섞여 있어서 해석에 주의를 기울 여야 했다. 수면장애가 전혀 없는 대상자로 $\mathrm{RBD}(-)$ 군을 구 성했어야 하나, 본 연구의 실험군과 연령과 성별을 맞춘 정 상군 자료 수집에 한계가 있었다. 단, 불면증을 주로 호소한 환자들은 RBD 병력이 없다고 해도 포함되지 않았다.

정리하면, 본 연구에서는 치료받지 않은 특발성 $\mathrm{RBD}$ 환 자의 주요 임상 문제인 꿈 회상 빈도와 수면의 질을 분석하였 다. $\mathrm{RBD}$ 환자의 수면의 질이 낫고, 꿈 회상 빈도가 높음에 도 불구하고, 수면의 질과 꿈 회상 빈도 및 잠꼬대와의 유의 한 상관성이 없음을 확인했다. 이는 $\mathrm{RBD}$ 환자가 신경퇴행 성 변화에 따른 수면에 대한 인지 오인을 갖고 있음을 시사 하는 중요한 근거가 될 것이다.

\section{Supplementary Materials}

The online-only Data Supplement is available with this article at https:// doi.org/10.13078/jsm.17008.

\section{REFERENCES}

1. Schenck CH, Mahowald MW. REM sleep behavior disorder: clinical, developmental, and neuroscience perspectives 16 years after its formal identification in SLEEP. Sleep 2002;25:120-138.

2. Schenck CH, Hurwitz TD, Mahowald MW. REM sleep behaviour disorder: an update on a series of 96 patients and a review of the world literature. J Sleep Res 1993;2:224-231.

3. Boeve BF, Silber MH, Ferman TJ, et al. REM sleep behavior disorder and degenerative dementia: an association likely reflecting Lewy body disease. Neurology 1998;51:363-370.

4. Boeve BF, Silber MH, Saper CB, et al. Pathophysiology of REM sleep behaviour disorder and relevance to neurodegenerative disease. Brain 2007;130(Pt 11):2770-2788.

5. Postuma RB, Gagnon JF, Vendette M, Fantini ML, Massicotte-Marquez J, Montplaisir J. Quantifying the risk of neurodegenerative disease in idiopathic REM sleep behavior disorder. Neurology 2009;72: 1296-1300.

6. Olson EJ, Boeve BF, Silber MH. Rapid eye movement sleep behaviour disorder: demographic, clinical and laboratory findings in 93 cases. Brain 2000;123(Pt 2):331-339.

7. Revonsuo A. The reinterpretation of dreams: an evolutionary hypothesis of the function of dreaming. Behav Brain Sci 2000;23:877-901; discussion 904-1121.

8. Hall C, Domhoff B. Aggression in dreams. Int J Soc Psychiatry 1963;9:
259-267.

9. Fantini ML, Corona A, Clerici S, Ferini-Strambi L. Aggressive dream content without daytime aggressiveness in REM sleep behavior disorder. Neurology 2005;65:1010-1015.

10. Fantini ML, Ferini-Strambi L, Montplaisir J. Idiopathic REM sleep behavior disorder: toward a better nosologic definition. Neurology 2005; 64:780-786.

11. Koulack D, Goodenough DR. Dream recall and dream recall failure: an arousal-retrieval model. Psychological Bulletin 1976;83:975-984.

12. Schredl M, Wittmann L, Ciric P, Götz S. Factors of home dream recall: a structural equation model. J Sleep Res 2003;12:133-141.

13. Schredl M, Bozzer A, Morlock M. Traumerinnerung und schlafstörungen [Dream recall in sleep disorders]. Psychother Psychosom Med Psychol 1997;47:108-116.

14. Berry RB, Brooks R, Gamaldo CE, et al. The AASM manual for the scoring of sleep and associated events. Rules, terminology and technical specifications. 2nd ed. Darien: American Academy of Sleep Medicine, 2012: 50-55.

15. American Academy of Sleep Medicine. International Classification of Sleep Disorders-third edition (ICSD-3). Darien: AASM Resource Library, 2014.

16. Lee HG, Choi JW, Lee YJ, Jeong DU. Depressed REM sleep behavior disorder patients are less likely to recall enacted dreams than non-depressed ones. Psychiatry Investig 2016;13:227-231.

17. De Gennaro L, Ferrara M, Cristiani R, Curcio G, Martiradonna V, Bertini M. Alexithymia and dream recall upon spontaneous morning awakening. Psychosom Med 2003;65:301-306.

18. Ji KH, Joo EY, Hong SB. Demographic, clinical and polysomnographic findings in 53 patients with rapid eye movement sleep behavior disorder. J Korean Sleep Res Soc 2012;9:41-45.

19. Arnulf I, Neutel D, Herlin B, et al. Sleepiness in idiopathic REM sleep behavior disorder and Parkinson disease. Sleep 2015;38:1529-1535.

20. Postuma RB, Gagnon JF, Pelletier A, Montplaisir JY. Insomnia and somnolence in idiopathic RBD: a prospective cohort study. NPJ Parkinsons Dis 2017;3:9.

21. Wu MN, Lai CL, Liu CK, et al. More severe hypoxemia is associated with better subjective sleep quality in obstructive sleep apnea. BMC Pulm Med 2015;15:117.

22. Trajanovic NN, Radivojevic V, Kaushansky Y, Shapiro CM. Positive sleep state misperception - a new concept of sleep misperception. Sleep Med 2007;8:111-118.

23. Wyatt JK, Bootzin RR, Allen JJ, Anthony JL. Mesograde amnesia during the sleep onset transition: replication and electrophysiological correlates. Sleep 1997;20:512-522.

24. Perlis ML, Smith MT, Orff HJ, Andrews PJ, Giles DE. The mesograde amnesia of sleep may be attenuated in subjects with primary insomnia. Physiol Behav 2001;74:71-76.

25. Sasai T, Matsuura M, Inoue Y. Electroencephalographic findings related with mild cognitive impairment in idiopathic rapid eye movement sleep behavior disorder. Sleep 2013;36:1893-1899.

26. Schredl M, Schäfer G, Weber B, Heuser I. Dreaming and insomnia: dream recall and dream content of patients with insomnia. J Sleep Res 1998;7:191-198.

27. Schredl M. Dreams in patients with sleep disorders. Sleep Med Rev 2009;13:215-221. 